\title{
Targeted Antagonism of Vascular Endothelial Growth Factor Reduces Mortality of Mice with Acute Respiratory Distress Syndrome*
}

\author{
Zhao ZHANG ${ }^{1}$, Dong-shi $\mathrm{LU}^{1}$, Dan-qing ZHANG ${ }^{1}$, Xin $\mathrm{WANG}^{2}$, Yu MING ${ }^{3 \#}$, Zhou-yang WU ${ }^{1 \#}$ \\ ${ }^{I}$ Department of Anesthesiology, Union Hospital, Tongji Medical College, Huazhong University of Science and Technology, \\ Wuhan 430022, China \\ ${ }^{2}$ Department of Senior Cadre, No. 989 Hospital, The Joint Logistics Support Force of the Chinese People's Liberation Army, \\ Luoyang 471003, China \\ ${ }^{3}$ College of Health Science and Nursing, Wuhan Polytechnic University, Wuhan 430032, China
}

(C) Huazhong University of Science and Technology 2020

\begin{abstract}
Summary: Acute respiratory distress syndrome (ARDS) is associated with a mortality of $45 \%$. Our previous research indicated that anti-vascular endothelial growth factor (VEGF) could maintain the normal structure and function of the respiratory barrier. However, systemic application of VEGF antagonists would lead to animal death. This study attempts to study the targeted drug delivery for ARDS. In this study, we used soluble fms-like tyrosine kinase-1 (sFlt)-targeted ultrasound microbubbles to antagonize the effect of VEGF on lung tissue. Ninety male BALB/ c mice were randomly assigned to 6 groups: phosphate buffer saline (PBS) group (PBS+PBS); blank group (PBS+empty microbubbles); lipopolysaccharide (LPS) group (LPS+PBS); ARDS group (LPS+empty microbubbles); control group (PBS + sFlt microbubbles); and treatment group (LPS + sFlt microbubbles). After administration of LPS or PBS in the corresponding groups, the sFlt-targeted microbubbles or empty microbubbles were injected into the blood circulation. Then the lungs were irradiated with ultrasound, which ruptured the drug-loaded microbubbles and helped release drugs to the lung tissues targeted. The lung injury score, lung wet/dry ratio (W/ D), liver and kidney functions, and the mortality of the mice in all groups were investigated at the predetermined time point. The difference in mortality between groups was examined by Fisher test. Other data were analyzed by one-way analysis of variance (ANOVA). A value of $P<0.05$ indicates that the difference was significant. The results showed that the $\mathrm{PaO}_{2}$ levels were normal in the PBS group, the blank group, and the control group. The LPS group and ARDS group showed significant hypoxia. $\mathrm{PaO}_{2}$ was improved significantly in the treatment group. The lung injury score and W/ D were normal in the PBS group, the blank group, and the control group. The lung injury score and W/D increased significantly in the LPS group and ARDS group and decreased in the treatment group $(P<0.05)$. The mortality rate of the ARDS model was $60 \%(95 \%$ confidence interval $47.5 \%-72.5 \%)$, and that with sFlt-targeted microbubbles was significantly lower at only $40 \%(95 \%$ confidence interval $27.5 \%-52.5 \%, P<0.05)$. It was concluded that anti-VEGF with sFlt targeted ultrasound microbubbles attenuated the lung injury and ultimately reduced the 7-day mortality effectively. It might be a suitable therapeutic tool for the treatment of ARDS.
\end{abstract}

Key words: acute respiratory distress syndrome; vascular endothelial growth factor; soluble fmslike tyrosine kinase-1; ultrasound microbubble; lipopolysaccharide

Acute respiratory distress syndrome (ARDS) is a common critical disease in clinic and is associated with a mortality of around $45 \%{ }^{[1]}$. Our previous studies indicated that anti-vascular endothelial growth factor

Zhao ZHANG, E-mail: lyzzaaaa@126.com

\#Corresponding authors, Zhou-yang WU, E-mail:wzy6638@, hotmail.com; Yu MING, E-mail: 497396197@qq.com

*This study was supported by Scientific Research Fund of Hubei Provincial Department of Education (No. B2016081) and Young Scholar Research Grant of Chinese Anesthesiologist Association (No. 21700007).
(VEGF) with soluble fms-like tyrosine kinase-1 (sFlt) could maintain the normal structure and function of respiratory membrane in lung ${ }^{[2,3]}$. However, it has been reported that systemic application of sFlt might reduce vascular permeability and affect the function of other organs such as liver and kidney, with clinical manifestations being anuria, malaise, or even death ${ }^{[4,5]}$. It appears that the adverse reaction of sFlt has restricted its clinical application. The approach as how to make sFlt target the lung may play an important role in the treatment of ARDS. 
Recent advancements in ultrasound microbubblemediated drug delivery technology has shown that this approach can improve spatially confined delivery of drugs to target tissues and reduce the toxicity ${ }^{[6,7]}$. In this study, sFlt was loaded into microbubble pockets and injected into animals. After the drug-loaded microbubbles entered the blood circulation, the lungs were irradiated with ultrasound, which ruptured the drug-loaded microbubbles and enabled drug release to occur in the lung tissues targeted. The lung injury score and mortality were measured to evaluate the benefits of sFlt-loaded ultrasound microbubbles.

\section{MATERIALS AND METHODS}

\subsection{Microbubble Preparation and Ultrasound Irradiation Conditions}

The sFlt-targeted microbubbles were prepared by incubating sFlt (soluble vascular endothelial cell growth factor receptor-1, 471-F1, R\&D Systems, USA) with a mixture of 1,2-distearoyl-sn-glycero-3phosphocholine (4 mg/mL), 1,2-distearoyl-sn-glycero3-phosphoethanolamine $(2 \mathrm{mg} / \mathrm{mL})$, and polyethylene glycol $(2 \mathrm{mg} / \mathrm{mL})$ at $37^{\circ} \mathrm{C}$ for $20 \mathrm{~min}^{[2,7]}$. The mixture was filled with perfluorobutane gas and then mechanically shocked for $45 \mathrm{~s}$ prior to being stayed for $5 \mathrm{~min}$ and rinsed for 3 times with PBS. The average particle size of the microbubbles and the concentration were measured using an optical microscope. Encapsulation efficiency (EE) of sFlt-targeted microbubbles was determined with a sFlt ELISA Kit (Mouse sVEGFR1/ Flt-1 DuoSet ELISA, DY471, R\&D Systems, USA). The absorbance $(A)$ developed in proportion to the amount of specific cytokine was recorded using an enzyme mark instrument (SPECTRAMAX 190, Molecular Devices Corporation, USA) at $450 \mathrm{~nm}$ wavelength. A standard curve was thus generated to calculate the concentrations of sFlt. The EE is defined as follows:

Drug EE $=$ (Total dosage in the system-Free dosage in the carrier)/Total dosage in the system $\times 100 \%$.

The clinical ultrasound system as described in the literature (Accusonic, Metron Medical Australia Pty. Ltd., Carrum Downs, Australia) was used to irradiate the chest of a mouse model ${ }^{[7]}$. The therapy ultrasound was delivered at $3 \mathrm{MHz}, 2 \mathrm{w} / \mathrm{cm}^{2}$, duty cycle $20 \%$ for $2 \mathrm{~min}$. Ultrasound irradiation of the lungs with this energy density is expected to rupture microbubbles and release drugs.

The microbubble solution of $1 \mathrm{~mL}$ was centrifuged at $4^{\circ} \mathrm{C}$ for $12000 \mathrm{r} / \mathrm{min}$, and $500 \mu \mathrm{L}$ supernatant was taken to determine free dosage in the carrier. The microbubbles of the precipitates were broken by irradiation with the above-mentioned ultrasonic intensity, and the drug dosage in microbubbles was measured. The two dosages were added to get the total dosage in the system, and then EE was calculated.

\subsection{Animals and Sample Treatment}

Ethical approval for this investigation was obtained from the Institutional Animal Care and Use Committee at Tongji Medical College, Huazhong University of Science and Technology (IACUC Number: 354). All animal experiments complied with the ARRIVE

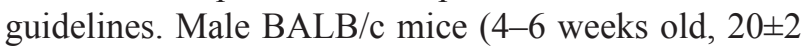
g) were obtained from the Animal Center of Tongji Medical College and the experiments were carried out in this center. The mice were kept in an animal facility for at least 3 days prior to experiments to allow adaptation to the environment and confirmation of their health. The animals were maintained on a lightdark cycle with light from 8:00 to 20:00 at $25^{\circ} \mathrm{C}$. Each animal received rodent laboratory chow and water $a d$ libitum. All experiments were approved by the animal care committee.

Ninety mice were randomly divided into 6 groups with 15 mice in each group: PBS group (PBS+PBS); blank group (PBS+empty microbubbles); lipopolysaccharide (LPS) group (LPS+PBS); ARDS group (LPS+empty microbubbles); control group (PBS+sFlt microbubbles); and treatment group (LPS + sFlt microbubbles). All mice were anesthetized with pentobarbital sodium $(40 \mathrm{mg} / \mathrm{kg}$, intraperitoneal injection). The mice in the LPS group, ARDS group, and the treatment group were intraperitoneally injected with $10 \mathrm{mg} / \mathrm{kg}$ LPS $(2 \mathrm{mg} / \mathrm{mL}$, Escherichia coli 055: B5, Sigma Chemical, USA) ${ }^{[8]}$. The mice in the PBS group, blank group, and the control group were intraperitoneally injected with equal volume of PBS. Right after LPS or PBS administration, sFlt microbubbles of $50 \mu \mathrm{g} / \mathrm{kg}(5 \mu \mathrm{g} / \mathrm{mL})$ were infused via the tail vein to each mouse in the control and the treatment group ${ }^{[9]}$. The mice in the PBS group and the LPS group were given equal volume of PBS. The mice in the blank and ARDS groups were given equal volume of empty microbubbles. The animals were then irradiated with chest ultrasound at $3 \mathrm{MHz}, 2 \mathrm{w} / \mathrm{cm}^{2}$, duty cycle $20 \%$ for 2 min to target the release of sFlt in lung tissue. The average treatment time per animal was about $4-5$ min.

Five mice were randomly selected from each group and the arterial blood samples were collected after $6 \mathrm{~h}$. Blood gas analysis was carried out with a blood gas analyzer (ABL700, Radiometer Co., Denmark). Glutamic-pyruvic transaminase (GPT) and blood urea nitrogen (BUN) were measured with an automatic biochemistry analyzer (AU640, OLYMPUSOPTICAL Co., Ltd., Japan) to evaluate the function of liver and kidney respectively ${ }^{[10]}$. Immediately after cardiac puncture, the mouse was killed by exsanguination and the thorax was opened with a midline thoracotomy. The right upper lobes of the lungs were harvested and immersed in $10 \%$ formalin until processing in paraffin 
wax for histopathological examination. The lung tissues of $0.5 \mathrm{~g}$ were made into homegenate, and the supernatants were frozen at $-20^{\circ} \mathrm{C}$. The right lower lobes of the lungs were fixed with glutaraldehyde and $4 \%$ phosphate solution mixture. The left lung was weighed (wet weight) using an electronic balance in a clean test tube and then dried in a constant temperature drying box $\left(80^{\circ} \mathrm{C}, 24 \mathrm{~h}\right)$. The dry weight was weighed and the wet weight to dry weight ratio (W/D) was calculated.

The remaining 60 mice were returned to their original feeding environment to allow feeding and drinking freely. The behavior and 7-day mortality of the mice were closely observed.

\subsection{Lung Injury Score and Electron Microscopic Examination of Lung Tissue}

The right upper lobes of the lung slides were examined with a light microscope. The lung injury score was recorded as described previously. The right lower lobes of the lung slides were examined by an electron microscope (Tecnai G2 12, FEI Co., Holland).

\subsection{VEGF Levels in Lung Tissue}

The VEGF level in the lung tissue homogenate was determined using an enzyme-linked immunosorbent assay kit for mouse VEGF according to the manufacturer's instruction (MMV00, R\&D Systems Incorporation, USA). The VEGF levels in different groups were measured and calculated as reported previously ${ }^{[2]}$.

\subsection{Statistical Analysis}

Statistical analysis was conducted using SPSS 23.0 for windows (SPSS Co., Ltd., USA). Data were expressed as means $\pm \mathrm{SD}$ except for lung injury scores which were represented as median (range). The lung injury scores were analyzed using the Kruskal-Wallis rank test followed by Mann-Whitney $U$ test. The difference in mortality between groups was examined by Fisher test. The other data were analyzed by oneway analysis of variance (ANOVA). A value of $P<0.05$ indicates that the difference was significant.

\section{RESULTS}

\subsection{Parameters of Microbubbles}

The light microscopy showed that the sFlttargeted microbubbles were uniform and the surface was smooth (fig. 1). The particle size ranged from 1.8 to $5.1 \mu \mathrm{m}$, with an average particle size of $2.9 \mu \mathrm{m}$.

Fig. 2 showed the standard curve of sFlt concentration and $(A)$ values. The EE of sFlt-targeted microbubbles was $89.73 \% \pm 6.85 \%$.

Fig. 3 showed that the experimenter injected drugloaded microbubbles into the tail vein of mice and monitored whether the microbubbles reached target organs.

There were no microbubbles in lung at $0 \mathrm{~s}$

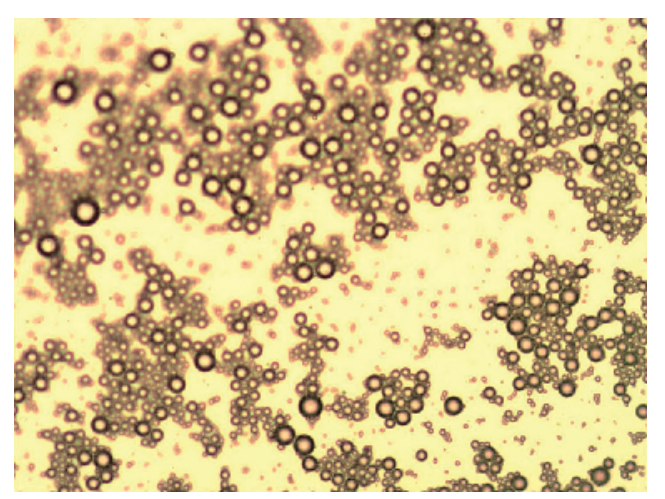

Fig. 1 sFlt-loaded microbubbles under a light microscope $(\times 40)$

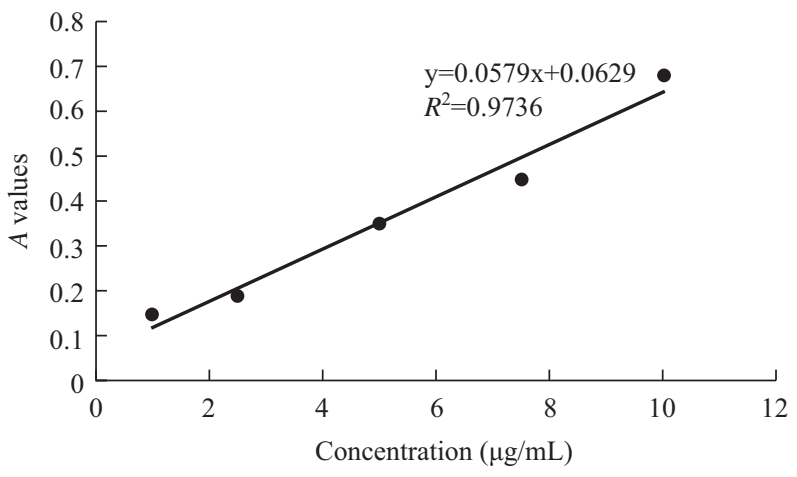

Fig. 2 Standard curve of sFlt concentration and absorbance $(A)$ values

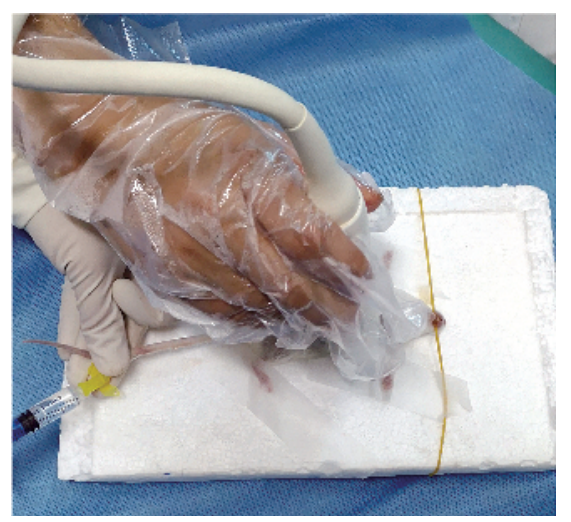

Fig. 3 Monitoring whether microbubbles reached the target organs after tail vein injection

The mouse was anesthetized and has been given LPS or PBS, then microbubbles were infused via tail vein.

(fig. 4A) after the tail vein injection of drug-loaded microbubbles, while a large number of microbubbles were observed at $71 \mathrm{~s}$ (orange dispersion point, fig. 4B). Through the next step of ultrasound irradiation, the drug-loaded microbubbles were released to lung tissue.

\subsection{Behavior, Organ Function, Lung Injury Score, W/D, and Mortality}

Mice in ARDS group and treatment group showed obvious erect hair, chills, malaise, shortness of breath, and then sleepiness clumps. They did not eat, their eyes were covered with secretions, and some mice 

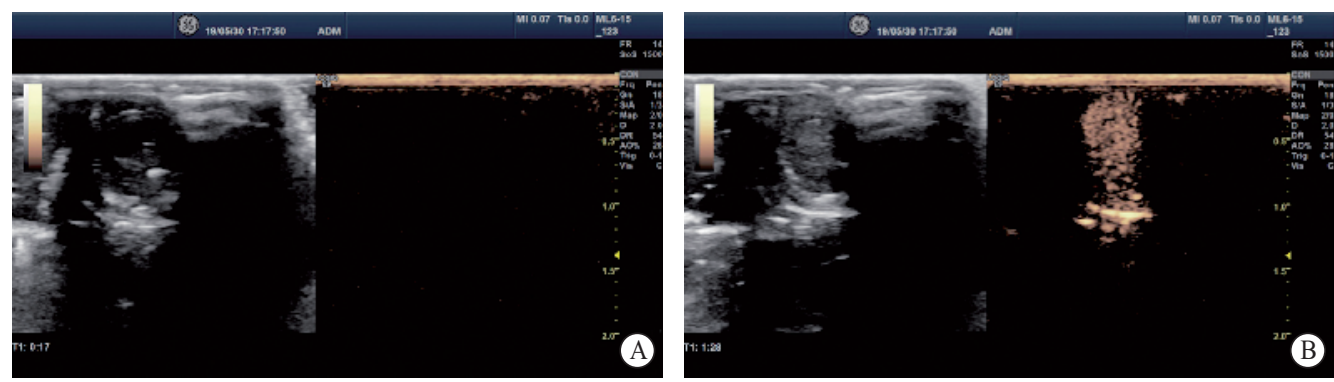

Fig. 4 A: Images of microbubbles injected at $0 \mathrm{~s}$ showed that microbubbles did not reach the target organ; B: Images of microbubbles injected at $71 \mathrm{~s}$ showed that microbubbles reached the target organ (orange dispersion point is the sound shadow formed by ultrasound microbubbles. The location of the ultrasound probe was in the thorax of the mice, and the orange dispersion point reflected that the ultrasound microbubbles entered the blood circulation through the heart. Ultrasound irradiation of drug-loaded microbubbles in lung tissue can release drugs in the target organs).

eventually developed cyanosis with slowed breathing.

Fig. 5 showed the organ functions in 6 groups examined in this work. There were no significant

7-day

A

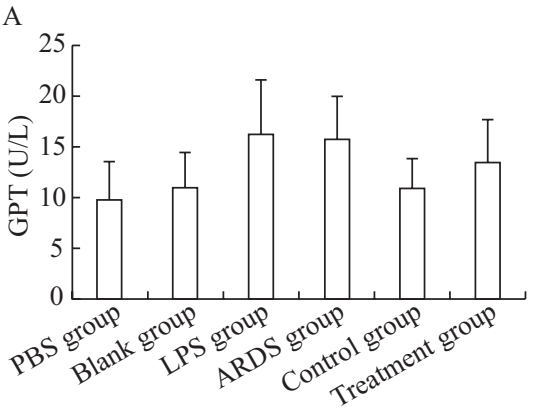

B

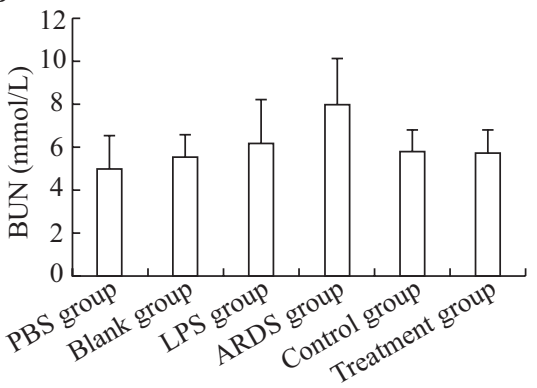

$\mathrm{E}$
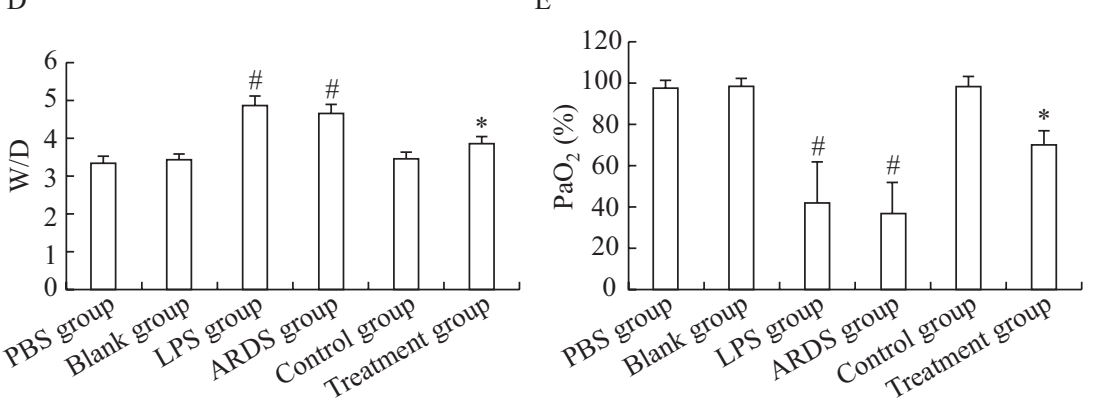

differences in the GPT and BUN among the 6 groups $(P>0.05$; fig. 5A and 5B). This suggested that the sFlt-targeted microbubbles had no significant adverse
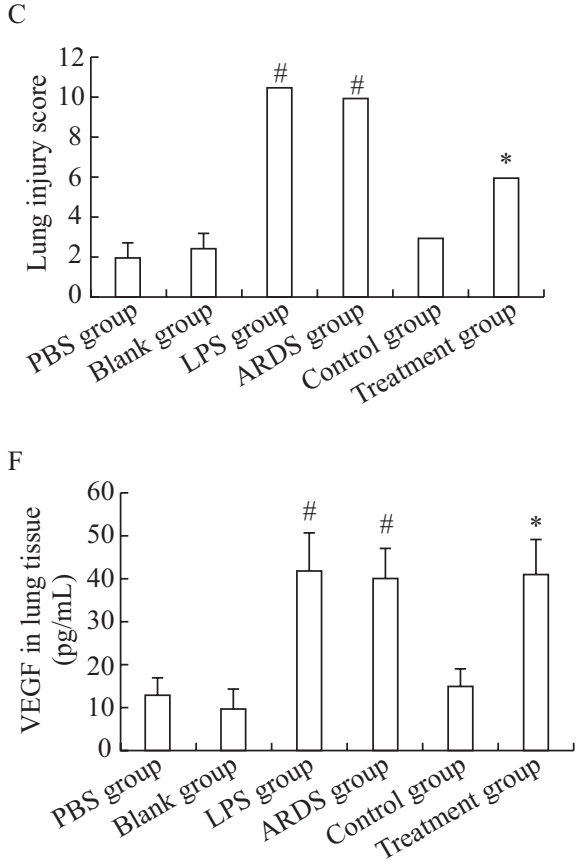

G

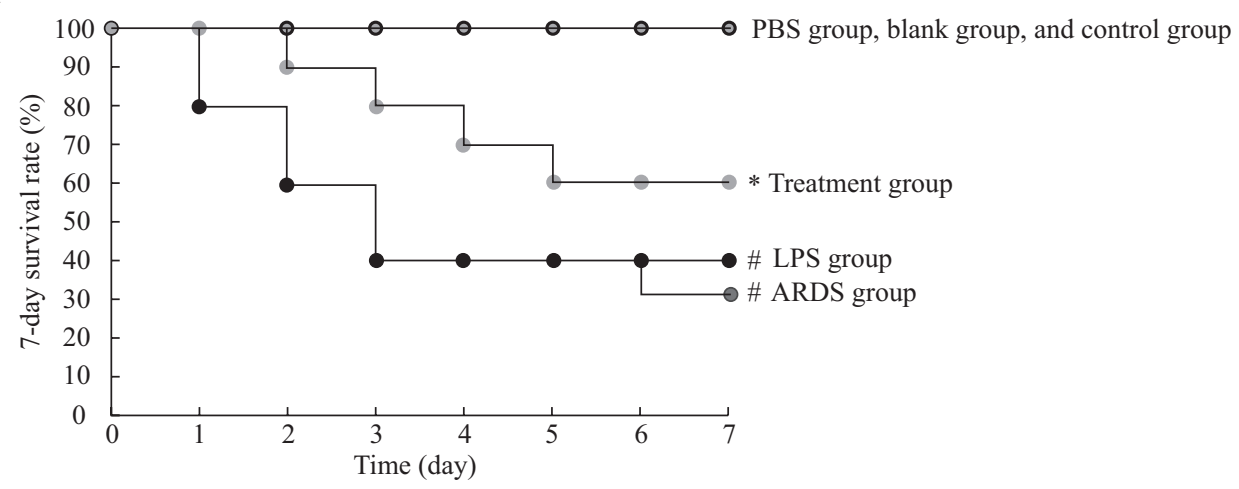

Fig. 5 Organ function and mortality in different groups

A: glutamic-pyruvic transaminase (GPT) levels in different groups; B: blood urea nitrogen (BUN) levels in different groups; C: comparison of the severity of lung injury score in each group; D: comparison of the wet weight to dry weight rate (WD) of lung tissue in different groups; E: the blood gas analysis in different groups; F: VEGF in lung tissue in different groups; G: 7-day survival rate in different groups. ${ }^{\#} P<0.05$ vs. blank group, ${ }^{*} P<0.05$ vs. ARDS group 
reaction on the liver and kidneys.

The mice in the PBS group, blank group, and the control group showed normal lung injury score and W/D (fig. 5C and 5D) and those in the LPS and ARDS groups exhibited significantly increased lung injury score and W/D. As compared with those in the ARDS group, mice in the treatment group presented decreased lung injury score and W/D $(P<0.05)$.

The $\mathrm{PaO}_{2}$ levels were normal in the PBS group, the blank group, and the control group (fig. 5E). The LPS group and ARDS group showed significant hypoxia $(P<0.05) . \mathrm{PaO}_{2}$ was improved significantly in the treatment group $(P<0.05)$.

As shown in fig. 5F, the VEGF levels in the LPS group, the ARDS group, and the treatment group were significantly higher than those in the PBS group, the blank group, and the control group $(P<0.05)$.
As shown in fig $5 \mathrm{G}$, the mortality rate of the ARDS model was $60 \%$ (95\% confidence interval $47.5 \%$ $72.5 \%$ ), and that with sFlt-targeted microbubbles was significantly lower at only $40 \%$ (95\% confidence interval $27.5 \%-52.5 \%, P<0.05$ ).

\subsection{Electron Microscopic Examination of Lung Tissue}

Fig. 6 showed the ultrastructure of lung tissue in different groups. The tight junction of lung tissue in the PBS group (fig. 6A), blank group (fig. 6B), and control group (fig. 6E) was closely connected and the respiratory membrane was thin. The tight junction in LPS group (fig. 6C) and ARDS group (fig. 6D) was widely loosened, the respiratory membrane was thickened, and leukocytes infiltrated into the lung tissue. In the treatment group (fig. 6F), the tight connection was basically normal, and the shape of
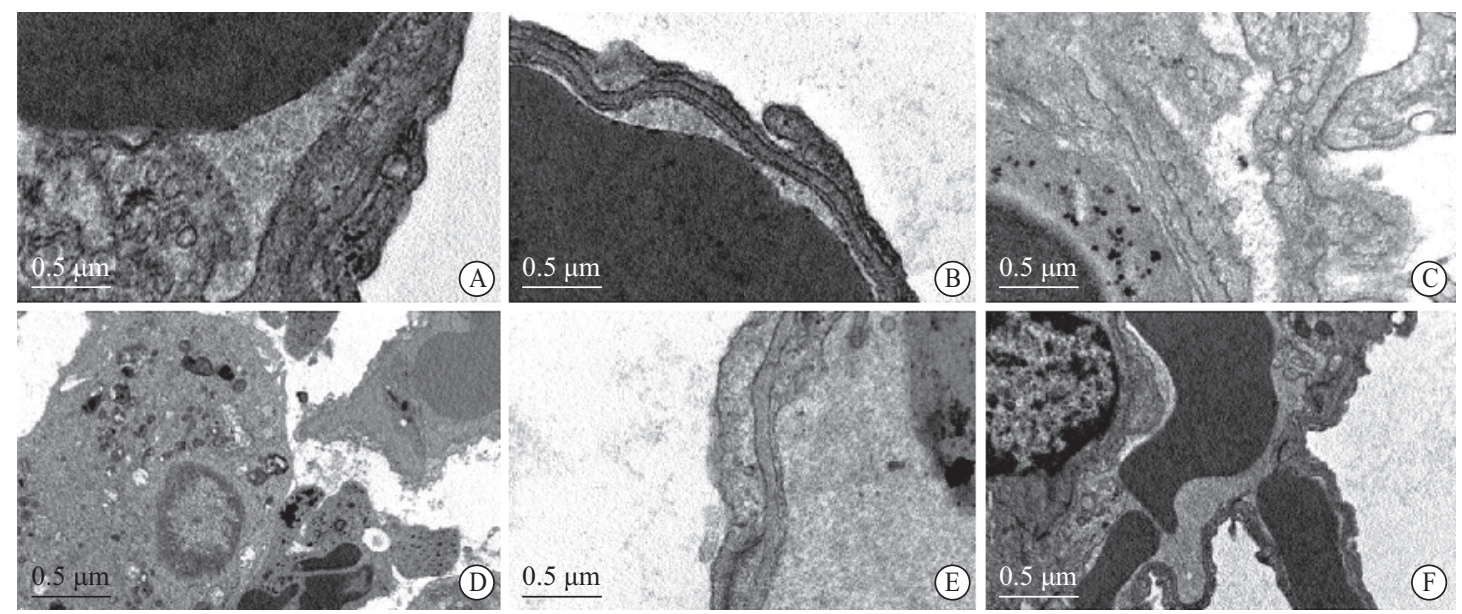

Fig. 6 Electron microscopic examination of lung tissue

A: PBS group; B: blank group; C: LPS group; D: ARDS group; E: control group; F: treatment group

respiratory membrane was relatively normal.

\section{DISCUSSION}

Our research indicated that LPS intraperitoneal injection brought about shortness of breath, cyanosis, and increased W/D and lung injury score. The ARDS model was characteristic of extensive morphological lung damage, such as edema, incrassation of the alveolar wall, and infiltration of inflammatory cells into alveolar and interstitial spaces ${ }^{[1]]}$. This model was fully consistent with the etiology, pathophysiology and clinical characteristics of ARDS as reported previously. In this study, the mortality rate of ARDS group was $60 \%$, which was slightly higher than the clinical mortality as reported by other researchers ${ }^{[12]}$. This will make it easier to study the effects of protective drugs.

In this study, sFlt-targeted microbubbles were stable and had high encapsulation efficiency ${ }^{[13]}$. After the drug-loaded microbubbles entered the blood circulation, the lungs were irradiated by ultrasound, which ruptured the drug-loaded microbubbles and enabled drug release to occur in the lung tissues targeted. The encapsulation and targeted release of microbubbles retarded liver and kidney damage resulting from direct systemic administration of $\mathrm{sFlt}^{14,}$ 15]. Moreover, sFlt-targeted microbubbles decreased pulmonary edema and maintained the normal structure and function of respiratory membrane in lung tissue. These pieces of evidence suggested that sFlt-targeted microbubbles reached the lung tissue accurately and blocked the effect of VEGF in the target organ.

This work indicated that the increased VEGF participated in the damage of three layer structure of respiratory barrier through tight junction proteins. In line with our previous studies, anti-VEGF targeted with sFlt-targeted microbubbles could decrease the loosening of tight connection. And sFlt-targeted microbubbles could decrease the lung injury score and pulmonary edema (W/D), repair the alveolar epithelial barrier, and improve the gas exchange function of ARDS mode ${ }^{[16-18]}$. As a result, the sFlt-targeted microbubbles 
could effectively improve the gas exchange function of lung.

Our previous study have shown that VEGF played an important role in the pathogenesis of inflammation ${ }^{[2]}$. Anti-VEGF with sFlt could maintain the normal structure and function of respiratory membrane in lung tissue via tight junction proteins. However, sFlt systemic application has been shown to reduce vascular permeability and affect the function of other organs, such as liver and kidney with clinical manifestations being anuria, malaise, and even death ${ }^{[14,15]}$. Therefore, in our previous studies, systemic application of sFlt did not reduce the mortality of animal models. In this study, we targeted the release of sFlt in lung tissue, which can effectively improve the mortality of ARDS animal model. This is the most important result of this study. This study makes sFlt closer to clinical application.

To sum up, our research indicates that anti-VEGF targeted with sFlt-targeted microbubbles works to maintain the normal function of the liver and kidneys and could be a suitable therapeutic tool for the treatment of ARDS.

\section{Conflict of Interest Statement}

I declare on behalf of my co-authors and myself that we do not have any conflict of interest.

\section{REFERENCES}

1 Beitler JR, Schoenfeld DA, Thompson BT. Preventing ARDS: progress, promise, and pitfalls. Chest, 2014, 146(4):1102-1113

2 Zhang Z, Wu Z, Xu Y, et al. Vascular endothelial growth factor increased the permeability of respiratory barrier in acute respiratory distress syndrome model in mice. Biomed Pharmacother, 2019,109:2434-2440

3 Tao H, Li N, Zhang Z, et al. Erlotinib Protects LPSInduced Acute Lung Injury in Mice by Inhibiting EGFR/ TLR4 Signaling Pathway. Shock, 2019,51(1):131-138

4 Wewers TM, Mayer AB, Pfleiderer A, et al. Increased soluble fms-like tyrosine kinase 1 after ischemia reperfusion contributes to adverse clinical outcomes following kidney transplantation. Kidney Int, 2019, 95(5):1091-1102

5 Wallace K, Bean C, Bowles T, et al. Hypertension, Anxiety, and Blood-Brain Barrier Permeability Are Increased in Postpartum Severe Preeclampsia/ Hemolysis, Elevated Liver Enzymes, and Low Platelet Count Syndrome Rats. Hypertension, 2018,72(4):946-
954

6 Xing L, Shi Q, Zheng K, et al. Ultrasound-Mediated Microbubble Destruction (UMMD) Facilitates the Delivery of CA19-9 Targeted and Paclitaxel Loaded mPEG-PLGA-PLL Nanoparticles in Pancreatic Cancer. Theranostics, 2016,6(10):1573-1587

7 Chen Z, Xie M, Wang X, et al. Efficient gene delivery to myocardium with ultrasound targeted microbubble destruction and polyethylenimine. J Huazhong Univ Sci Technolog Med Sci, 2008,28(5):613-617

8 Wu Z, Ming Y, Zhang Z, et al. Edaravone rescues the lung by inhibiting lipid peroxidation and proinflammatory cytokines in a rat model. Chin Med J (Engl), 2014,127(5):968-970

9 Zebrowski BK, Yano S, Liu W, et al. Vascular endothelial growth factor levels and induction of permeability in malignant pleural effusions. Clin Cancer Res, 1999,5(11):3364-3368

10 Ibrahim KE, Al-Mutary MG, Bakhiet AO, et al. Histopathology of the Liver, Kidney, and Spleen of Mice Exposed to Gold Nanoparticles. Molecules, 2018, 23(8):1848-1850

11 Franco RD. Mouse Models of Acute Lung Injury and ARDS. Methods Mol Biol, 2018,1809:341-350.

12 Giacomo B, John GL, Tài P, et al. Epidemiology, Patterns of Care, and Mortality for Patients with Acute Respiratory Distress Syndrome in Intensive Care Units in 50 Countries. JAMA, 2016,23;315(8):788-800

13 Liao AH, Li YK, Lee WJ, et al. Estimating the delivery efficiency of drug-loaded microbubbles in cancer cells with ultrasound and bioluminescence imaging. Ultrasound Med Biol, 2012,38(11):1938-1948

14 Mahasreshti PJ, Kataram M, Wang MH, et al. Intravenous delivery of adenovirus-mediated soluble FLT-1 results in liver toxicity. Clin Cancer Res, 2003,9(7):2701-2710

15 Leelahavanichkul A, Huang Y, Hu X, et al. Chronic kidney disease worsens sepsis and sepsis-induced acute kidney injury by releasing High Mobility Group Box Protein-1. Kidney Int, 2011,80(11):1198-1211

16 Cockrell AS, Yount BL, Scobey T, et al. A mouse model for MERS coronavirus-induced acute respiratory distress syndrome. Nat Microbiol, 2016,28;2(2):1622616230

17 Smith BJ, Grant KA, Bates JH. Linking the development of ventilator-induced injury to mechanical function in the lung. Ann Biomed Eng, 2013,41(3):527-36

18 Gotts JE, Bernard O, Chun L, et al. Clinically relevant model of pneumococcal pneumonia, ARDS, and nonpulmonary organ dysfunction in mice. Am J Physiol Lung Cell Mol Physiol, 2019,317(5):L717-L736

(Received Sept. 25, 2019; accepted May 14, 2020) 